\title{
Comparative Study of Various Transformations in Robust Watermarking Algorithms
}

\author{
S.Poongodi, \\ Assistant Professor \\ K.S.R. college of engineering, \\ Tiruchengodu.
}

\author{
B.Kalaavathi, PhD. \\ Prof \& Head, Dept. of CSE, \\ K.S.R. Institute for engineering \& Technology, \\ Tiruchengodu.
}

\begin{abstract}
In the digital world, that is currently evolving at such a rapid pace, intellectual copyright protection is becoming increasingly important. This is due to digital data being particularly simple to copy and resell without any loss of quality. Digital representation and distribution of data has increased the potential for misuse and theft and thus gives rise to problems associated with copyright protection and the enforcement of these rights. The main technical approaches to address the challenge of intellectual copyright protection are digital watermarking techniques.
\end{abstract}

The spread spectrum technology has become an important technique that is not only widely used for still image and video watermarking, but also used for audio watermarking.A robust watermarking algorithm to watermark JPEG2000 compressed and encrypted images.

The encryption algorithm is proposed to use a stream cipher. While the proposed technique embeds watermark(the transmission of question paper in online for a secure manner) in the compressed-encrypted domain, the extraction of watermark can be done in the decrypted domain.

Based upon this application a study made for this transformation such as multiwavelet, contourlet and lifting scheme decomposition and also compare the characteristics of these transformations using LSB technique for various file formats and the spread-spectrum watermarking (SSW) technology. Experimental results show the various characteristics of different transforms, the embedding capacity,robustness, perceptual quality and security of the proposed algorithm, and their efficiency with various image formats in watermarking using LSB algorithm and spread spectrum.

Key words: Multiwavelets, contourlet, lifting scheme, Spread spectrum, SSW, LSB, PSNR, NC

\section{INTRODUCTION}

Watermarking is a process of hiding an object to a multimedia element. These are considered as image, audio and video. Watermarking is widely used in multimedia application and secret data transmission etc. Since the digital data has no difference in quality between the original and its copy, it is impossible to distinguish original from the copy [1].Data can be embedded and extracted or detected in multimedia element.

Many innovative ideas have emerged in the field of digital image processing due to the invention of wavelets. Since the wavelet transform is a reversible process, it has been effectively and successfully used in many areas such as image compression, pattern recognition etc. Even though it has many advantages, it also has some drawbacks such as limited directional representation, anisotropicity etc.

The coefficients that are obtained using the wavelet transform could be used for watermarking. Digital watermarking is a technique of embedding a watermark in a multimedia data using certain type of algorithms that are suitable for processing intellectual property rights. There are two types of watermarking based upon the human visual system; visible watermarking and invisible watermarking. This paper deals with invisible watermarking technique, since security is the major consideration. Invisible watermarking is more robust and more secure than the visible watermarking; it can be used for copyright protection or authentication. The embedding locations are unknown and only the users who have the secret keys in the watermarked image can extract the secret image.

Watermark should be robust against the image processing operations like filtering, re-quantization, dithering, scaling, cropping etc. Watermarking algorithms can be broadly classified into two categories based upon embedding the data into images; spatial domain and transform domain. Spatial domain watermarking is the earliest developed technique which provides lower bit capacity. Transform domains can embed large number of bits. This paper uses the LSB algorithm and spread spectrum for watermarking algorithms. This solution also provides robustness to the "analog hole," or rerecording with a separate device (e.g. using a camcorder pointed at a computer screen to record video playback). This solution also provides robustness to collusion attacks.

The current implementation of this technology provides a forensic solution, in which each media provider marks each copy of a piece of content with a watermark specific to the consumer of the content (e.g. a person, a distributor, a theater, etc.). In this case the provider can track pirated content back to the initial consumer.

A media provider could also further develop this watermarking technology to embed information into media files and prevent playback of content by users without sufficient rights.As a solution to copyright protection problems, many digital watermarking schemes have been proposed for intellectual property right protectionof digital media data, in which one of the most promising techniques is that called spread-spectrum watermarking (SSW) technology [1]-[11].

The SSW is a technique that uses a concept from spreadspectrum communication and embeds the watermark represented by pseudorandom sequence as noise patterns into signals in spatial (time) domain or frequency domain. One of the important characteristics of pseudorandom sequences is its 
correlation property, i.e., the autocorrelation calculated between the pseudo- random sequences generated by the same initiator has the maximalvalue. On the contrary, the crosscorrelation calculated between pseudorandom sequences generated by different initiators has a very small value. Therefore, the watermark detectionfor SSW technology can be completed by computing the correlations between pseudorandom sequences and watermarked signal in spatial (time) domain or frequency domain, and comparing the correlations to a threshold to determine whether watermarkis present in the signal.

Another techniques for watermarking is the least-significant bit (LSB) modification.LSB algorithm uses the least significant bits of the pixels of the cover image for embedding the watermark.

In the LSB algorithm, the security information was hidden in the cover image by replacing the LSB bits. These bits are not significant because it is not robust against attacks. If the binary information with the length of $\mathrm{s}$ is to be hidden, it is necessary to select s blocks from $M \times N$ matrix first. Then a pixel of every block is chosen to hide the binary information .The LSB algorithms could be classified into sequential, random and array etc, based upon the method of bit selection. The sequential embedding approach is the simplest.

There are distinct difference of the statistical characters between the embedding part and other part of the image. It has the lowest security. To overcome this disadvantage, random and array approaches replace the sequential approach gradually. Because the secret information hidden in cover image in a random manner and so the information is distributed everywhere. The embedded and not embedded part cannot be distinguished. The array approach decreased the changing amount of unimportant bits. The advantages of LSB algorithm is that it has huge information capacity, quick embedding speed, and can be hidden and extracted easily. Hence the LSB algorithm is used in all the three different transformations and the results are analyzed in this paper.

In this paper video watermarking and the overview of proposed scheme are discussed in section II. Multiwavelets are discussed in section III, Contourlet in section IV, Lifting Scheme in section $\mathrm{V}$, the performance of these transformations are analyzed, results have been discussed in the section VI and section VII concludes.

\section{VIDEO WATERMARKING}

Video watermarking involves embedding cryptographic information derived from frames of digital video into the video itself.

Ideally, a user viewing the video cannot perceive any difference between the original, unmarked video and the marked video, but a watermark extraction application can read the watermark and obtain the embedded information.

This watermarking algorithm is optimized to achieve three separate factors:

1. Robustness: the ability of the watermark to resist attempts by an attacker to destroy it by modifying the size, rotation, quality, or other visual aspects of the video.

2. Security: the ability of the watermark to resist attempts by a sophisticated attacker to remove it or destroy it via cryptanalysis, without modifying the video itself.
3. Perceptual fidelity: the perceived visual quality of the marked video compared to the original, unmarked video.

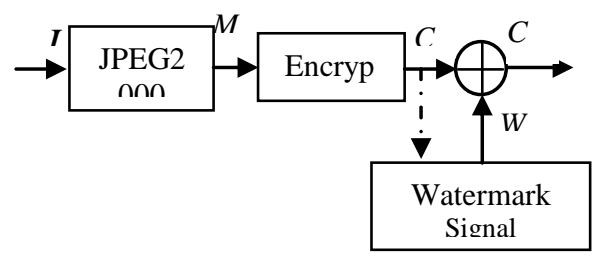

(a)

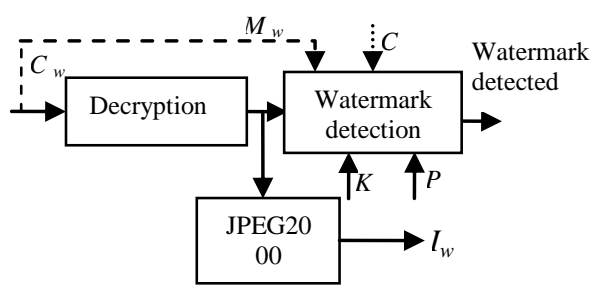

(b)

Fig.1 a) Watermark embedding and

\section{b) Watermark extraction}

The proposed algorithm works on JPEG2000 compressedcode stream. JPEG2000 compression is divided into fivedifferent stages [18]. In the first stage the input image is preprocessed by dividing it into non-overlapping rectangular tiles. The unsigned samples are then reduced by a constant tomake it symmetric around zero and finally a multi-component transform is performed. In the second stage, the discrete wavelet transform(DWT) is applied followed by quantization in the third stage. Multiple levels of DWT gives a multiresolution image. The lowest resolution contains the low-pass image while the higher resolutions contains the high-pass image.

These resolutions arefurther divided into smaller blocks known as code-blocks where each code-block is encoded independently. Further, the quantized-DWT coefficients are divided into different bit planes and coded through multiple passes at embedded block coding with optimized truncation (EBCOT) to give compressed byte stream in the fourth stage. The compressed byte stream is arranged into different wavelet packets based on resolution, precincts,components and layers in the fifth and final stage. Thus, it is possible to select bytes generated from different bit planes of different resolutions for encryption and watermarking. The proposedalgorithm uses a symmetric stream cipher with additive homomorphic properties for encryption. In fact the distributors get JPEG2000compressed stream cipher encrypted images fordistribution. The distributors can then apply any robust additivewatermarking technique to this compressed encrypted stream.In this paper, the watermarking schemes, namely,Spread Spectrum (SS),LSB are used.

\section{MULTIWAVELETS}

Wavelets are useful for signal processing applications such as image compression and denoising. 
Multiscale representation is generated by using Multiwavelets transform. The main difference between the Multiwavelets and conventional wavelet is that in multiwavelet each channel in the filter bank has a vector valued input and vector valued output because the energy and entropy features are extracted easily. A scalar valued input signal must be converted into a suitable vector valued signal. This conversion is called preprocessing. In Multiwavelet transform the image is decomposed into four subbands. TheMultiwavelet iterates only the low frequency components. The sub bands are the result of decomposition of low frequency components. The decomposition involves pre-filtering of rows and columns. Pre-filtering takes place by using critically sampled and oversampled schemes. In the oversampled scheme the rows and columns are repeated. As a result of repetition redundancy is added, which is desirable for feature extraction[1].
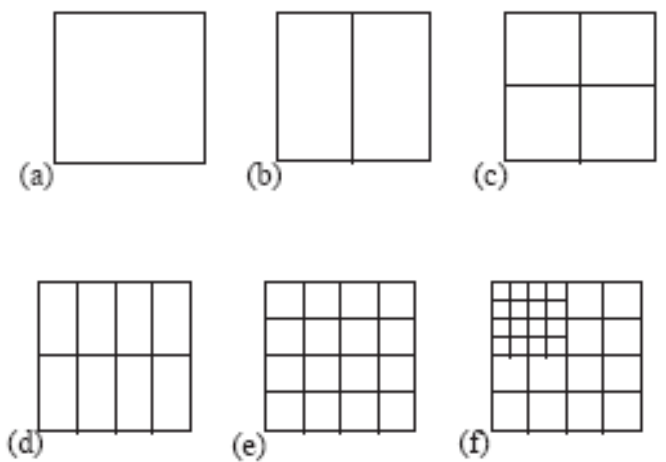

Fig 2: a)Original image, b)After pre-filtering along rows, c)After pre-filtering along columns, d)First level decomposition along rows, e)First level decomposition along columns, f)Second level decomposition.

Critical sampling does not add redundancy and therefore is most suitable for applications such as compression.Fig 2 and Fig 3 show the Multiwavelets decomposition

Multiwavelet can simultaneously provide perfect reconstruction while preserving length (orthogonality),good performance at boundaries(via linear phase symmetry) and higher order of approximation(vanishing moments).In this paper the most well-known Multiwavelets named GHM, which was constructed by Geronimo, Hardin and Massopust is used.GHM scaling functions have short support and symmetry property[2]. Multiwavelets offer the possibility of superior performance for image processing applications when compared to conventional wavelets.

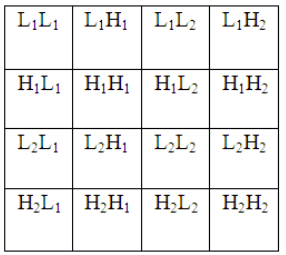

(a)

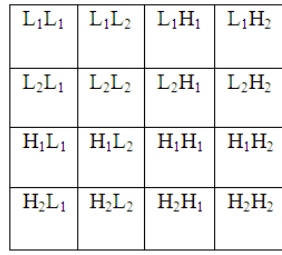

(b)
Fig3:a)Multiwavelets output

b)After rearranging the output.
Here the watermark is embedded in the middle frequency since it contains equal number of noise and data.

\section{CONTOURLET:}

Contourlet transform is a recently developed transform technique to overcome the drawbacks of the wavelets and Curvelets. Due to the good non-linear approximation performance for piecewise smooth functions in one dimension, wavelets have a growing impact in the field of signal processing.

Wavelets are good at representing the point discontinuities which are generated by edges, where the edges correspond to the sharp transitions in the intensity [3].

The images with two dimensional piecewise smooth functions will have one dimensional discontinuities. Even though the wavelets in two dimensions are good at capturing the discontinuities along the edge points they failed to represent the discontinuities along the smooth contours as they do not exhibit the properties like directionality and anisotropicity. To overcome these disadvantages curvelet transforms was introduced [3].

The curvelet transform was initially constructed in the continuous domain and then discretised for sampled data but this became a challenge to possess all the features promised by curvelet in the discrete domain as in the continuous domain, especially when critical sampling is desirable.

Also the curvelet transform was clearly defined only in the frequency domain and sampling of the curvelets was not clear in the spatial domain.

All the above facts motivated to propose a filter bank structure which can deal with the piecewise smooth images with smooth contours effectively. The result is a frame composed of contour segments, named as contourlets. Initially this transform was developed in the discrete domain and then it is translated to the continuous domain via the multiresolution analysis framework and iterated filter bank structure[4]. The contourlet transform has the following advantages over the other transforms:

1) It provides sparse representation for piecewise smooth images with smooth contours.

2) It has localization and multiresolution features.

3) It has directionality and anisotropicity.

In this transform, to obtain the sparse image expansion, multiscale transform is applied. In the multiscale transform, the nearby wavelet coefficients are grouped since they are locally correlated .

Then to gather those nearby basis functions at the same scale into linear structures, a local directional transform is applied. Multiscale transform is used for edge detection and the local directional transform is used for contour segments detection.To implement this contourlet transform, double filter bank structure is used. This structure is a combination of LP (Laplacian pyramid) and DFB (directional filter bank) and it is said to be Pyramidal directional filter bank as shown in Fig.4. 


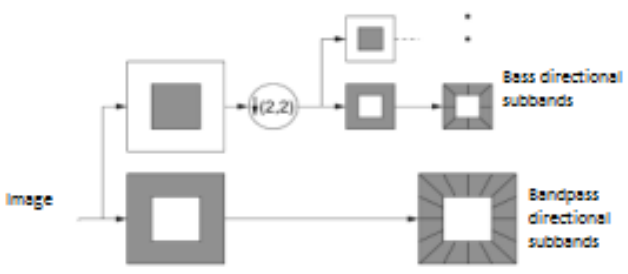

Fig.4 Double Filter Bank Structure

The edge detection is performed by the Laplacian pyramid and the contour segments detection is performed by the DFB. LP captures the point discontinuities which results in a band pass image. The DFB links those edge points into contour segments. In the contourlet transform each generation doubles the spatial resolution as well as angular resolution. Here the watermark is embedded in the mid-frequency band. The Laplacian pyramid and the frequency partition of DFB are shown in Fig.5 and Fig.6:

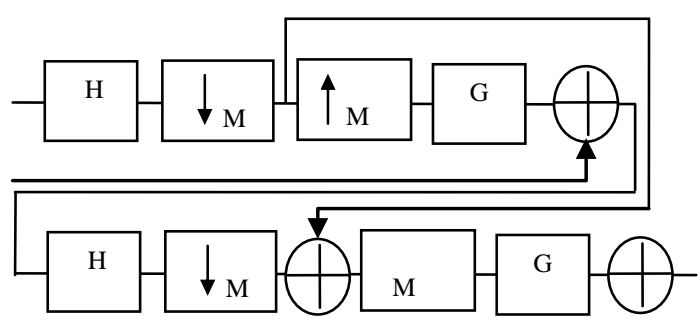

Fig.5 Laplacian Pyramid

The LP decomposition at each level generates a downsampled low pass version of the original and the difference between the original and the prediction, resulting in a bandpass image.

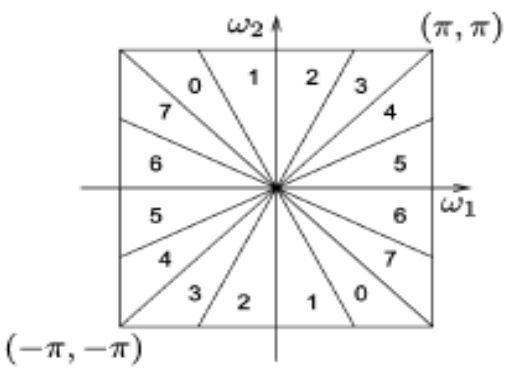

Fig.6Frequency Partition of DFB

The DFB is efficiently implemented via a L-level tree structured decomposition that leads to two to the power of L subbands with wedge shaped frequency partition as shown in Fig.6.

\section{LIFTING SCHEME:}

The lifting scheme is an efficient method to implement wavelet transform. The traditional wavelet is based on Fourier transform, translation and dilation and it is termed as First generation wavelets. The first generation wavelets are not suitable for curved surfaces. The lifting scheme is a second generation method to construct the biorthogonal wavelet which inherits the MRA of the first generation wavelet. It is easy to implement and needs less storage and hence could be realized using DSP chips. Also integer wavelet transform can be easily implemented using lifting scheme.
Lifting scheme algorithms have the advantage that they do not require temporary arrays in the calculations steps and have less computations. Fig.7 shows the block diagram of the lifting scheme [6].

\subsection{Three Steps in lifting scheme [8]}

a)Split step

It is also called lazy wavelet transform. It divides the input data into odd and even elements.

b)Predict step

This step predicts the odd elements from the evenelements.

c)Update step

This replaces the even elements with an average.

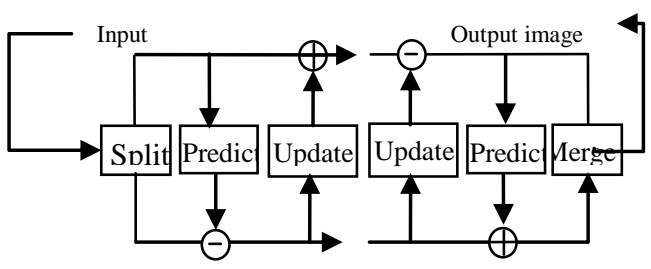

Fig.7 Lifting scheme.

There are three steps in lifting scheme: split, predict, and update. In the first step, the original signal $\mathrm{S}$ is decomposed into low frequency signal $\mathrm{S}$ and high frequency signal $\mathrm{D}$. Secondly a predictive operator $\mathrm{P}$ operates on $\mathrm{S}$ to modify the high frequency details as shown below.

$$
S_{j+1}^{\prime}=S_{j+1}+U\left(D_{j+1}\right)
$$

where

$S_{j+1}^{\prime}$ is the low frequency coefficients of wavelet transform[5].

The lifting scheme can be accomplished through iterating the original signal. The reconstruction is accomplished using the reverse process. Here the watermark is embedded in the midfrequency sub band.

\section{RESULTS AND DISCUSSION:}

This study tested the methods of Multiwavelets, Contourlet, Lifting schemes and spread spectrum for the transmission of data using watermarking method through video signals. Comparison is done with the parameters of PSNR, Normal Correlation (NC) and Root Mean Square Error (RMSE).These parameters are taken at the transmission of watermarking data through video signal transmission The watermark being embedded in the mid-frequency sub band of the original image is decomposed by the various transforms. The resultant images for the question paper information are compared with one another using different metrics.

\section{METRICS USED:}

PSNR EQUATION:

$$
\frac{\left.(m * n) * \max (\max (x(i, j)))^{2}\right)}{\sum_{i=0}^{m} \sum_{j=0}^{n}\left((x(i, j)-y(i, j))^{2}\right)}
$$




\section{NORMALCORRELATION EQUATION:}

$$
\frac{\sum_{i=0}^{m} \quad \sum_{j=0}^{n}(P(i, j)-Q(i, j))}{\sum_{i=0}^{m} \sum_{j=0}^{n}(P(i, j))^{2}}
$$

\section{ROOT MEAN SQUARE ERROR:}

$$
\frac{1}{K} \sum_{i=1}^{K}(\hat{S}-S)^{2}
$$

where,

$\mathrm{m}, \mathrm{n}$ are the height and width of the input image, $\mathrm{X}$ represents the cover image and $\mathrm{Y}$ represents the embedded image. P and Q represent the original and extracted watermark respectively.

$S$ represents original image and $\hat{S}$ represents embedded image. $\mathrm{K}$ is the image size.

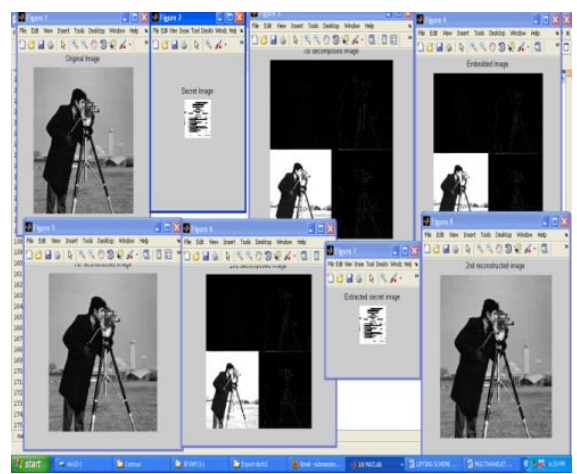

Fig. 8 shows the original cover image and decomposition of the contourlet scheme

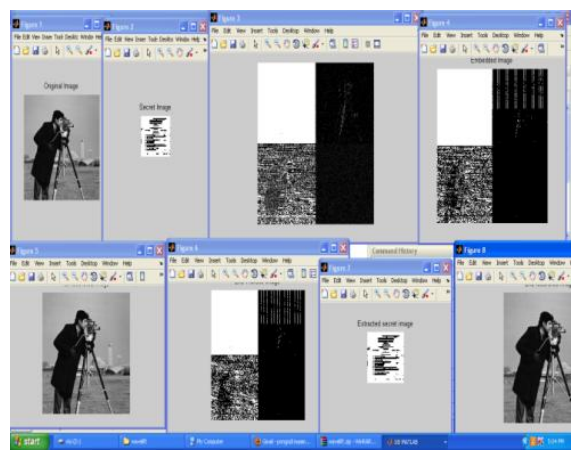

Fig. 9 shows the original cover image anddecomposition of lifting scheme

The fig. 8 and fig. 9 shows that the decomposition levels of contourlet scheme and lifting scheme. The decomposed image consists of original cover image,input image,watermarked image.

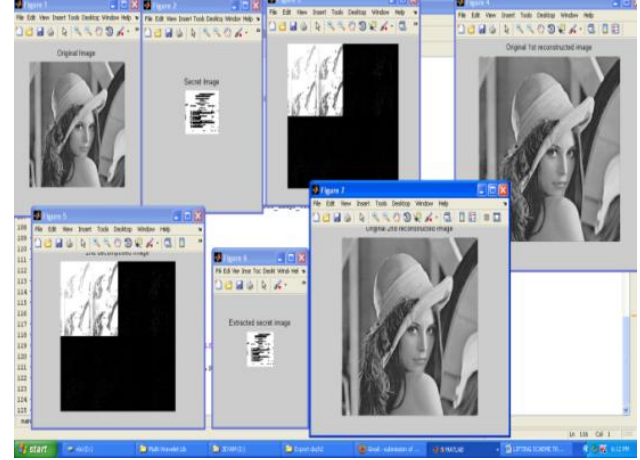

Fig.10 shows the original cover image and decomposition of the multiwavelets,

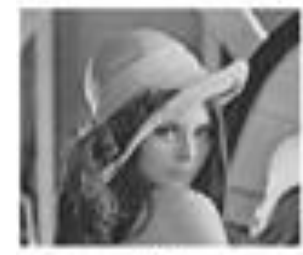

(a)

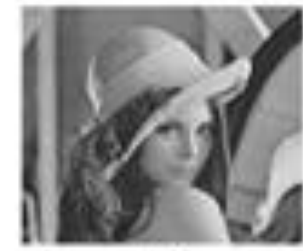

(b)

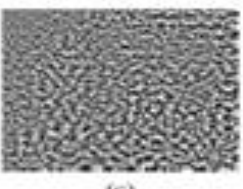

(s)

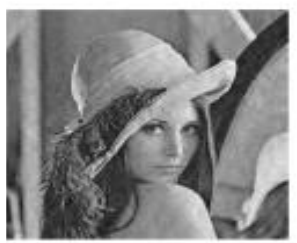

(d)

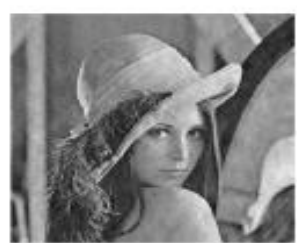

(e)
Fig. 11 shows the spread spectrum technique-(a) Original image. (b) Unwatermarked-decompressed image (47.8 dB). (c) Encrypted image. (d)Watermarked image (resolutions 1, 2, and 3) (35.22 dB). (e) Watermarked image (all resolutions) $(32.58 \mathrm{~dB})$.

Table1: Comparison of diffenert transformation with MATLAB result

\begin{tabular}{|c|l|c|c|}
\hline $\begin{array}{c}\text { INPUT } \\
\text { IMAGE }\end{array}$ & $\begin{array}{c}\text { TRANSFORMATI } \\
\text { ONS }\end{array}$ & $\begin{array}{c}\text { PSNR( } \\
\mathbf{d b})\end{array}$ & NC \\
\hline \multirow{3}{*}{ LENA } & Multiwavelet & 33.5 & 1 \\
\cline { 2 - 4 } & Contourlet & 32.8 & 1 \\
\cline { 2 - 4 } & Lifting scheme & $\mathbf{3 7 . 7}$ & 1 \\
\hline \multirow{2}{*}{$\begin{array}{c}\text { CAMER } \\
\text { AMAN }\end{array}$} & Multiwavelet & 33.53 & 1 \\
\cline { 2 - 4 } & Contourlet & 30.98 & 1 \\
\cline { 2 - 4 } & Lifting scheme & $\mathbf{3 6 . 8}$ & 1 \\
\hline CROWD & Multiwavelet & 29.8 & 1 \\
\hline
\end{tabular}




\begin{tabular}{|c|l|c|c|}
\hline & Contourlet & 28.7 & 1 \\
\cline { 2 - 4 } & Lifting scheme & $\mathbf{3 1 . 7}$ & 1 \\
\hline LENA & $\begin{array}{l}\text { Spread } \\
\text { spectrum }\end{array}$ & $\mathbf{3 8 . 4 5}$ & $\mathbf{1}$ \\
\hline
\end{tabular}

The table gives the comparision analysis between Multiwavelet, Contourlet,Lifting scheme and spread spectrum methods using different input images and different file formats. Among this the spread sectrum method give better PSNR value compared to the remaining transformations. This shows that the spread spectrum method is suitable for the secure transmission of on line question paper transmission without any loss. In reception side, the question paper is retrieved exactly as same as transmission side. Because the normal correlation value is 1 .

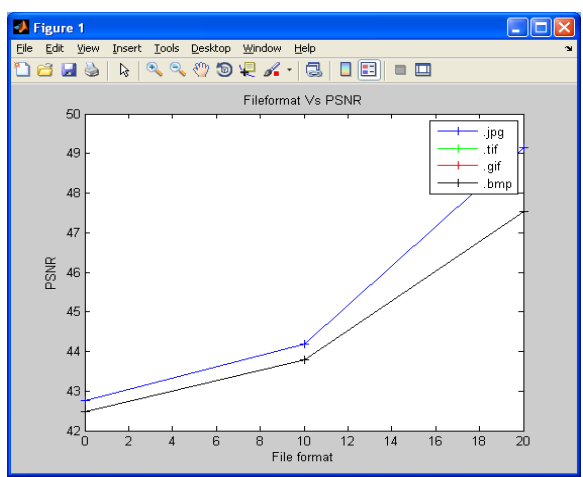

Fig.12 Comparision performance between multiwavelet, Countorlet and lifting scheme for all test images

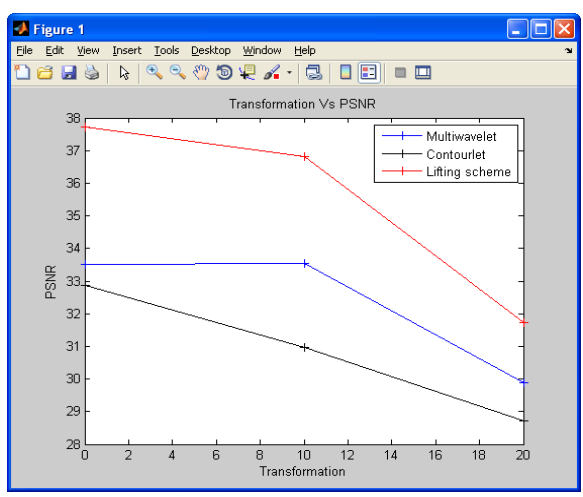

Fig.13 Comparision performance between multiwavelet, Countorlet and lifting schemes for PSNR with different file format of the test images

In fig. 12 and fig. 13 shows that the comparision result between multiwavelet,contourlet and lifting scheme methods using different file formats of an image.This shows that the differnet file formats of an images does not change the PSNR value.

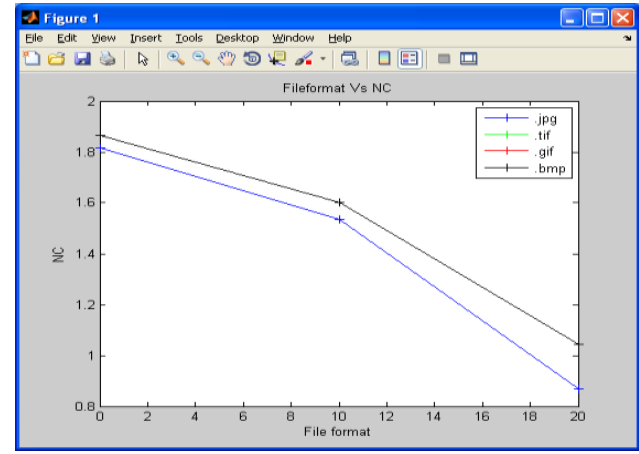

Fig.14 Comparision performance between multiwavelet, Countorlet and lifting schemes for $\mathrm{NC}$ with different file format of the test images

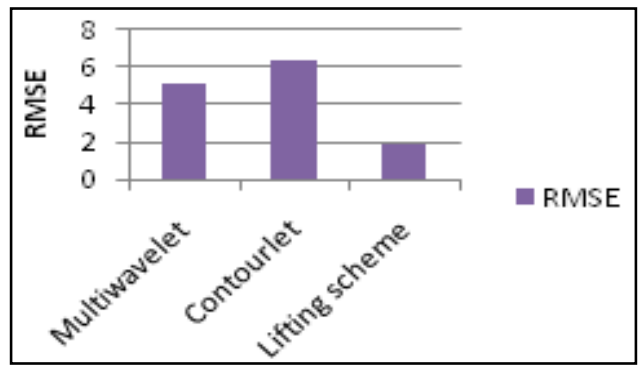

Fig.15 Root Mean square value comparison of multiwavelet,contourlet and lifting scheme

In fig. 15 shows that the root mean square value of multiwavelet, contourlet and lifting scheme.The RMSE value helps to predict the difference between original cover image and retrieved cover image after transformations. Whenever the difference between original image and retrieved image is low that method gives better performance than other transformation.

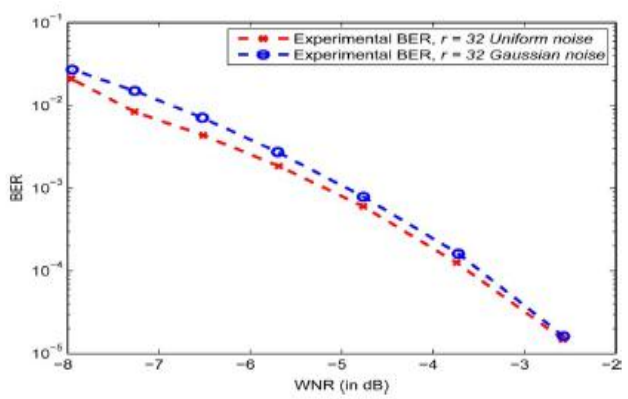

Fig.16 BER versus WNR with $r=32$ for SS watermarking scheme (nonblind detection).

\section{CONCLUSION:}

In the video watermarking technique proposed for on line question paper data transmission in a secure manner. For this application, multiwavelet, contourlet, lifting scheme and spread spectrum methods have been compared with different metrices. These matrices is tested for different types of images and different file formats.Based upon the performance analysis of various transformations with different file formats, 
the PSNR ratio of the spread spectrum technique is greater than the other transformations in the video watermarking.so the spread spectrum technique give high secure transmission of online question paper transmission effectively.

\section{REFERENCES}

[1] Hamid soltanian-zadeh, Farshid RafieeRad,SiamakpoulabdollahnejadD' Comparision of multiwavelet, wavelet,haralick and shape features for microcalcification classification in mammograms'2004 pattern recognition society, published by Elsevier Ltd.

[2] Yuanhaishao, Wei chen, Chan liu 'Multiwavelets-based digital watermarking with support vector machine Technique 'Proceedings of control and decision conference,2008.CCDC 2008.chinese 2-4 july 2008 page(s):4557-45561

[3] M. N. Do and M. Vetterli. 'The Contourlet transform: An efficient directional multiresolution image representation'. IEEE Trans. on Image Processing, 14(12):2091-2106, Dec. 2005

[4] M. N. Do and M. Vetterli,, 'Contourlet: a directional multiresolution image representation' Proceedings of ICIP, vol. 1, 2002, pp. 357-360.

[5] Matej Kseneman1, Dušan Gleich1, Peter Planinšič, VladoKitanovski and DimitarTaskovski 'Comparison between Different Lifting Scheme Algorithms' Proceedings of systems,signals and image processing,2008 IWSSIP 2008.15 ${ }^{\text {th }}$ international conference on 25-28 June 2008.page(s): 327-330.

[6] Zheng-weishen, Wei-wei Liao, Ya-nan shen 'Blind watermarking algorithm based on henon chaos system and lifting scheme wavelet'Proceedings of the 2009 International Conference on Wavelet Analysis and Pattern Recognition, Baoding, 12-15 July 2009

[7] Jinsheng Xiao, Benshun Yi, Yuan Yao 'Wavelets based on lifting scheme and its application on image Processing' Proceedings of 2008 International Symposium on Knowledge Acquisition and Modeling.

[8] A.AliceBlessie, J. Nalini and S.C.Ramesh, "Image Compression Using Wavelet Transform Based on the Lifting Scheme and its Implementation" International
Journal of Computer Science Issues IJCSI, Vol. 8, Issue 3, No. 1, May 2011,page(s) : 1694-0814.

[9] F. Battisti, M. Cancellaro, G. Boato, M. Carli, and A. Neri, "Joint watermarkingand encryption of color images in the Fibonacci-Haar domain,"EURASIP J. Adv. Signal Process., vol. 2009.

[10] M. Cancellaro, F. Battisti, M. Carli, G. Boato, F. De Natale, and A.Neri, "A joint digital watermarking and encryption method," in Proc. SPIE Security, Forensics, Steganography, and Watermarking of Multimedia Contents X, 2008, vol. 6819, pp. 68 191C-68 191C.

[11] J. Prins, Z. Erkin, and R. Lagendijk, "Anonymous fingerprinting withrobust QIM watermarking techniques,” EURASIP J. Inf. Security, vol.2007.

[12] Z. Li, X. Zhu, Y. Lian, and Q. Sun, "Constructing secure contentdependentwatermarking scheme using homomorphic encryption," inProc. IEEE Int. Conf. Multimedia and Expo, 2007, pp. 627-630.

[13] Q. Sun, S. Chang, M. Kurato, and M. Suto, "A quantitive semi-fragileJPEG2000 image authentication system," in Proc. Int. Conf. ImageProcessing, 2002, vol. 2, pp. 921924.

[14] R. Rivest, A. Shamir, and L. Adleman, "A method for obtaining digitalsignatures and public-key cryptosystems," Commun.ACM, vol. 21, no.2, pp. 120 126, 1978.

[15] S. Goldwasser and S. Micali, "Probabilistic encryption," J. Comput.Syst. Sci., vol. 28, no. 2, pp. 270-299, 1984.

[16] T. ElGamal, "A public key cryptosystem and a signature scheme basedon discrete logarithms," IEEE Trans. Inf. Theory, vol. 31, no. 4, pp.469-472, Jul. 1985.

[17] P. Paillier, "Public-key cryptosystems based on composite degreeresiduosity classes," Lecture Notes in Computer Science, pp. 223-238,1999.

[18] M. Rabbani and R. Joshi, "An overview of the JPEG 2000 still imagecompression standard," Signal Process. Image Commun., vol. 17, no.1, pp. 3-48, 2002.716 